\title{
Jane Addams y Eleanor Clarke Slagle: política, equidad social y ocupación desde los inicios de la Terapia Ocupacional
}

\author{
Jane Addams and Eleanor Clarke Slagle: politics, social equity \\ and occupation from the beginning of Occupational Therapy
}

\author{
Rodolfo Morrison J. ${ }^{1}$ \\ Recibido: 10 de agosto 2015 - Enviado para modificación: 20 de noviembre 2015 - Aceptado: 1 de diciembre 2015 \\ Morrison, R. (2015). Jane Addams y Eleanor Clarke Slagle: política, equidad social y ocupación desde los inicios de la Terapia \\ Ocupacional. Revista Ocupación Humana,15(2), pp. 5-17.
}

\begin{abstract}
Resumen: El objetivo del artículo es describir y reflexionar sobre algunas ideas y perspectivas de dos mujeres relevantes en el origen de la Terapia Ocupacional: Eleanor Clarke Slagle, y su mentora, Jane Addams. Ambas, a principios del siglo XX, desarrollaron acciones y constructos teóricos relacionados con la igualdad de género, la política y la justicia social; además, trabajaron juntas en un asentamiento social denominado Hull House, centro de gran influencia en Estados Unidos, principalmente por expandir el poder de las mujeres y por participar como generador de políticas a favor de la erradicación de las diferencias de clase y género. Este trabajo explora la vida de Clarke Slagle, sus relaciones con Addams y su fuerte lucha respecto a "lo social", presente en los inicios de la disciplina. Se evidencia como Addams y su enfoque en la equidad social, incluyendo la igualdad de género y el derecho a desempeñar diversas ocupaciones, fueron una piedra edificante para la Terapia Ocupacional que forjó Clarke Slagle, junto con otros pioneros de la disciplina.
\end{abstract}

Palabras clave: Historia, Terapia Ocupacional, feminismo.

Abstract: The aim of this paper is to describe and reflect on some of the ideas and perspectives of two important women relevant to the origins of Occupational Therapy: Eleanor Clarke Slagle, and her mentor, Jane Addams. In the early twentieth century, both developed actions and theoretical constructs related to gender equality, political and social justice, also both worked together on a social settlement called Hull House, a center of great influence in the United States, primarily for expanding women's empowerment and participating in crafting public policies in favor of the eradication of class and gender differences. This paper explores the life of Slagle, her relationship with Addams and her fierce struggle to address social issues, present at the beginning of the discipline. It makes evident how Addams and her focus on social equily, including gender equality and the right to participate in various occupations, were a milestone for Occupational Therapy. They were forged by Slagle along with other pioneers of the discipline.

Key words: History, Occupational Therapy, feminism.

\footnotetext{
${ }^{1}$ Licenciado en Ciencias de la Ocupación, Terapeuta Ocupacional. Doctor y Máster en Lógica y Filosofía de la Ciencia. Académico y coordinador, Área Investigación, Departamento de Terapia Ocupacional y Ciencia de la Ocupación, Facultad de Medicina, Universidad de Chile. Sociedad Chilena de Ciencia de la Ocupación. Comité de Estudios en Ciencia de la Ocupación. Núcleo I+D Gestión para la Diversidad, Área de Trabajo Social, Universidad de Chile. rodolfomorrison@med.uchile.cl - morrison.rodolfo@gmail.com
} 


\section{Introducción}

"El terapeuta ocupacional (...) debe mirar el pasado y al futuro. Del pasado adquiere la razón de ser, del futuro recibe una invitación para aplicar su herencia a colaborar en el desarrollo de una nueva sociedad". (Guillette, 1973, p. 51).

Este artículo sintetiza y propone diversas reflexiones del autor sobre algunas perspectivas iniciales de la Terapia Ocupacional, en donde se destacan conceptos como política, justicia social e igualdad de género. Para ello, se presenta parte de la vida de Eleanor Clarke Slagle y algunas de sus relaciones y construcciones teóricas efectuadas gracias a los fundamentos teóricos de Jane Addams.

Así, un aspecto relevante es considerar que la historia de la Terapia Ocupacional presentaría más de una versión (Morrison, 2011a). En una se expondría una perspectiva en la cual los varones son los generadores del conocimiento y de la fundación de la disciplina, y en otra, versión alternativa, se identificaría como esta fue generada, difundida y posicionada por mujeres.

Esta afirmación se sustenta en los estudios feministas sobre la ciencia (González y Pérez, 2002), que permiten una posición para criticar la historia "oficial" por considerarla androcéntrica y alejada de "la realidad de los hechos". Además, se plantea desde esta perspectiva que el conocimiento ha sido validado y construido solo por varones, incluso, por un grupo reducido de ellos, a saber: blancos, cristianos, heterosexuales y de clase media (Haraway, 1991), lo que genera maneras particulares y perspectivas ses- gadas de validar lo que entendemos por "realidad".

Una historia que no es justa con las mujeres y un conocimiento que no considera sus puntos de vista, son representaciones sesgadas de la realidad, lo que impide una concepción más amplia, justa y objetiva de lo que observamos y conocemos.

Desde este posicionamiento epistemológico feminista se establece que una de las figuras más relevantes en la construcción de la Terapia Ocupacional fue Eleanor Clarke Slagle. Ella habría sido una piedra angular en el desarrollo de la disciplina, debido a que contribuyó con fuertes fundamentos teóricos empleados hasta hoy por las y los terapeutas ocupacionales, y por expandir la profesión formando profesionales y posicionándola en centros hospitalarios (Kielhofner, 2009; Metaxas, 2000; Morrison, 2011a; 2011b; Sanz y Rubio, 2011).

Los fundamentos de Slagle provendrían de diferentes corrientes teóricas como el tratamiento moral, el movimiento de artes y oficios, el movimiento de higiene mental y el pragmatismo (Morrison, 2014). Estas perspectivas se consolidaron en lo que se conoce por Terapia Ocupacional, a raíz de un curso tomado por Slagle en la Hull House en 1911 (Breines, 1986; Quiroga, 1995). En este curso, Slagle se vincularía más de cerca con la Hull House y con las ideologías de las decenas de mujeres que trabajaban allí. Una de ellas, la cofundadora de la Hull House, Jane Addams.

Jane Addams fue la primera mujer del continente americano en ganar el Pre- 
mio Nobel de Paz, en 1931, y es reconocida como una de las cien mujeres más influyentes de todos los tiempos (Kuiper, 2010); desarrolló una política social fundamentada en el pragmatismo y en la igualdad de género, que influyó a Slagle (Breines, 1986; Knight, 2005).

A continuación se revisa parte de las vidas de Slagle y Addams, se presenta un esbozo de su importante trabajo, sus desarrollos teóricos, y cómo estos posicionaron conceptos como política, equidad social y ocupación desde los inicios de la disciplina, por lo que son parte inherente de la formación de terapeutas ocupacionales.

\section{Eleanor Clarke Slagle. Breve biografía y relaciones con la Hull House}

Eleanor Clarke Slagle nació en Hobart, Nueva York, en 1871. Fue la menor de dos hermanos, sus padres fueron WiIliam John Clarke y Emmaline J. Clarke (su apellido de soltera era Davenport) (James, 1971; Quiroga, 1995; Metaxas, 2000; Kielhofner, 2009; Encyclopædia Britannica, 2011; Sanz Valer y Rubio Ortega, 2011).

Emmaline Davenport se casó a los 16 años de edad con William Clarke, arquitecto de 36 años, después de que este retornara como segundo teniente de la Infantería de Nueva York durante la Guerra Civil en Estados Unidos. Tuvieron a su primer hijo, John, en 1896, y dos años después a su segunda hija, antes de que Emmaline cumpliera 20 años (Quiroga, 1995; Sanz y Rubio, 2011).

Según la historiadora Virginia M. Quiroga (1995), la infancia de Clarke Sla- gle fue similar a la de otras niñas y niños de su época, marcada por una tradicional división de los roles de género; su padre, un hombre estricto y frío, inculcaba reglas y responsabilidades, mientras su madre, con tono suave, consentía a sus hijos.

Clarke Slagle estudió música entre 1885 y 1886 en Claverack College. El objetivo de esa escuela no era inculcar valores cívicos de responsabilidad o participación (características por las cuales se destacaría en su adultez), sino educar en "maneras refinadas, carácter social elevado, un gusto culto y valores cristianos" (Sanders, citado por Quiroga, 1995, p. 43); ello hace suponer que gran parte de sus valores sobre la responsabilidad social provendrían de su familia.

Historiadores e historiadoras relatan que Clarke Slagle no dejó documentos relacionados con su vida personal, por lo que existen vacíos sobre su adolescencia, su vida de casada y la época previa a su vinculación con la Hull House (Quiroga, 1995).

Pese a lo anterior, se sabe que se casó con Robert E. Slagle en 1894. Robert era hijo del reverendo Peter y la señora Cordelia Slagle, ambos de Chicago. Se desconoce en qué trabajaba Robert para vivir, cómo se conocieron, si su matrimonio fue feliz, si tuvieron hijos, por qué vivieron en San Luis o por qué se divorciaron (Quiroga, 1995). Así pues, no se tienen datos sobre los 17 años que Eleanor estuvo casada con Robert, ni cómo habría llegado en 1911 a la Escuela de Cívica y Filantropía de Chicago (Chicago School of Civics and Philantropy), por 
ello, es difícil identificar con precisión las influencias que la llevaron a ser una líder de la Terapia Ocupacional (James, 1971).

En un documento de 1934, Clarke Slagle señaló que desde hace algunos años había estado interesada en la injusticia social hacia las personas con discapacidad física y enfermedad mental, debido a las influencias del profesor Henderson, de la Universidad de Chicago, así como de Jane Addams y Julia Lathop, de la Hull House, quienes habrían sido su inspiración para implicarse aún más en las reformas de salud mental, bajo el Movimiento de Higiene Mental.

Pese a lo anterior, algunas autoras y autores, entre ellos Quiroga (1995), Metaxas (2000) y Kielhofner (2009), refieren que la familia de Clarke Slagle, y sus condiciones de salud, ejercieron una fuerte influencia sobre ella, impulsándola a interesarse en un método que permitiera a las personas con algún tipo de incapacidad participar en actividades sociales y lograr una mejor calidad de vida.

Clarke Slagle estuvo rodeada de familiares muy cercanos con enfermedades que reducían su capacidad para desenvolverse en la sociedad. En ocasiones fue la "vigilante" de la familia y su experiencia le sirvió para estar preparada para el trabajo con personas con discapacidad física o mental (Quiroga, 1995).

Su padre regresó de la Guerra Civil con un grado de inmovilidad parcial, producto de un disparo en el cuello (Sanz Valer y Rubio Ortega, 2011). Su hermano John contrajo tuberculosis en su juventud y habría desarrollado adicción al alcohol, impulsado por diferentes problemas emocionales; ella luchó junto a él intentando resolverla, hasta que este falleció en un accidente automovilístico en 1933.

El hijo de John, Jack, sobrino de Clarke Slagle, contrajo poliomielitis en 1908 a la temprana edad de dos años, lo que limitó la movilidad de su brazo derecho, reduciendo a su vez sus habilidades para desempeñar las actividades de su vida diaria. Durante los años veinte, John puso a Jack bajo la tutela de su hermana, mientras él trabajaba en el Congreso de Estados Unidos como abogado (Quiroga, 1995).

Las experiencias con su padre, su hermano y su sobrino habrían sido las razones por las cuales Clarke Slagle buscó una manera de ayudar a personas con dificultades. Bajo los fuertes mandatos de género de su época, necesitó un nuevo significado para "ganarse la vida" después de su separación, por lo que tales experiencias fueron fundamentales para que dedicara su vida a la Terapia Ocupacional (Quiroga, 1995, p. 45).

Clarke Slagle, mientras trabajaba en el hospital de Kakankee (Illinois), habría observado los efectos nocivos de la inactividad, surgiendo su preocupación por el uso de artes y oficios en el tratamiento de personas con enfermedad mental (Sanz Valer y Rubio Ortega, 2011).

En 1911 comenzó a interesarse en la reforma de salud impulsada por el Movimiento de Higiene Mental, tomando el curso Curatives occupations and recreations (Ocupaciones curativas y 
recreación) en la Escuela de Cívica y Filantropía de Chicago, perteneciente a la Hull House y dictado por Julia Lathrop (1858-1932) (Metaxas, 2000; Kielhofner, 2009; Sanz Valer y Rubio Ortega, 2011). La metodología de este curso fue muy innovadora para la época, las estudiantes aprendían teóricamente por medio de lecturas y recibían entrenamiento en oficios, además de experiencia en la práctica clínica (Quiroga, 1995).

Fue entonces cuando comenzó a vincularse cercanamente con la Hull House, que para ese tiempo era el motor de la reforma social en Estados Unidos y fue centro del feminismo por décadas. En ese lugar muchísimas mujeres, lideradas por Jane Addams, introdujeron a la cultura estadounidense la posibilidad de un cambio en la estructura de la sociedad; uno de sus conceptos estaba vinculado a la igualdad en el desempeño de diferentes ocupaciones por parte de la sociedad, lo que puede relacionarse con el concepto contemporáneo de Justicia Ocupacional (Townsend y Wilcock, 2004).

\section{Antecedentes de la formación de Eleanor Clarke Slagle como terapeuta ocupacional}

Clarke Slagle se formó como terapeuta ocupacional en un ambiente muy particular, producto de diferentes revoluciones de las cuales podemos identificar tres: sociales, políticas y epistemológicas (Breines, 1986; Quiroga, 1995; Morrison, 2011a; 2011b;).

Primero, el movimiento feminista, entendido como la segunda ola que comienza a principios del siglo XX, corresponde a una revolución social que buscaba reivindicar y posicionar el rol de las mujeres en el espacio público (Freedman, 2003). Las mujeres durante años habían permanecido en sus hogares al cuidado de sus hijos y orientadas a un rol secundario en la sociedad; en aquel entonces exigieron salir a la calle, manifestarse y argumentar cómo sus cualidades "naturales" para el cuidado y el servicio debían ser puestas en práctica a favor de la sociedad (Quiroga, 1995). Exigían igualdad frente a sus pares varones y manifestaban que no soportarían más que sus opiniones no fueran escuchadas.

Una segunda revolución corresponde a lo político. Las fuertes diferencias de clase presentes en los inicios de 1900 en Estados Unidos, generaron varios cuestionamientos a las reformas laborales. Lo anterior, sumado a la potente entrada de inmigrantes provenientes principalmente de Europa y a la segunda revolución industrial (18701914, aprox.), provocó que las políticas públicas fueran puestas en tela de juicio por el movimiento obrero. En este sentido, y debido a que los trabajadores laboraban más de doce horas al día, se buscaron diferentes alternativas para "reparar" su salud mental. Es así como se fortalecen el Movimiento de Artes y Oficios y la Higiene Mental, ambos orientados a mejorar la salud de las personas (Addams, 1895). Julia Lathrop, trabajadora social de la Hull House, habría propuesto a Clarke Slagle cómo aplicar estas disciplinas en los hospitales (Quiroga, 1995).

Una tercera revolución corresponde a lo epistemológico. Durante el siglo XIX la ideología que explicaba la mayo- 
ría de los fenómenos alrededor era el racionalismo fundamentalista bajo un enfoque positivista (Echeverría, 1998). Se establecía una verdad objetiva, externa al ser humano, comprobable, única y universal; así como un conocimiento positivo, lineal y acumulable, del cual se podía dar cuenta a través de la mente (la que estaría separada del cuerpo) (Marcos, 2010). A fines del siglo XIX se comienza a cuestionar esta manera de comprender la ciencia y la realidad, uno de esos cuestionamientos provenía del pragmatismo.

El pragmatismo señala de qué manera la verdad será relativa a los observadores de acuerdo a los métodos que se empleen para mirar, indica cómo no es posible escindir al objeto del sujeto y explica cómo la mente no está separada del cuerpo. El pragmatismo rompe con los cánones tradicionales de verdad entregando a las ideas validez y verdad, de acuerdo a su utilidad en la sociedad (Addams, 1902; Pierce, 1907; James, 1909; Dewey, 1916). Desde esta perspectiva la verdad es variable y constantemente cambiante, de acuerdo a los requerimientos de la sociedad y no sólo de los métodos que se empleen, los cuales no tendrían la característica de ser objetivos per se.

En medio de estas tres revoluciones, Clarke Slagle se formó como terapeuta ocupacional en la Hull House de Jane Addams.

\section{Jane Addams y la Hull House}

Jane Addams fue sin duda una mujer excepcional. Su fuerte causa a favor de la cohesión social estaba fundamentada en la ideología pragmatista que inundaba los círculos intelectua- les de Estados Unidos (Knight, 2005). Desde la perspectiva frente a la cual nuestras ideas son "representaciones mutables de la realidad" (James, 1909), Addams creaba "realidades" en la práctica que luego eran "teorizadas", o fundamentadas o descritas en términos teóricos y viceversa, en una reciprocidad constante, recursiva, sistémica y circular (Addams, 1895; 1902; 1912a; 1916). Esto era, como el pragmatismo lo señala, integrar la teoría y la acción desarrollando una filosofía aplicada inmersa en la acción social, involucrando una perspectiva de género y de clases sociales, un tipo de pragmatismo mucho más radical que el expuesto por otros teóricos (Hamington, 2006).

Addams (1902; 1916) comprendía que las desigualdades sociales representaban aprendizajes y hábitos arraigados en la sociedad, los cuales con esfuerzo podrían ser modificados. Sin embargo, éstos no podían ser impuestos por un externo sin conocer la realidad y el concepto de verdad de quienes se pretendía ayudar; de este modo, criticó la labor de ciertos settlement workers (trabajadores de la caridad) que suponían conocer o preconcebir las necesidades y carencias de la gente, manifestando su creencia en una "moral superior", y que en muchas ocasiones culpaban a las mismas personas por su situación de pobreza (Addams, 1902).

Addams mantuvo la convicción de que las personas y las comunidades son capaces de crear nuevas realidades para mejorar su calidad de vida; refería que la fuerza de la democracia es inimaginable y que en su camino 
muchas veces son necesarios diferentes intentos para evidenciar sus beneficios. Lo anterior implica que las acciones deben analizarse por sus aspectos permanentes o transitorios y que, en ocasiones, pueden darse situaciones desagradables (malos entendidos, disputas), necesarias para la consecución de derechos y triunfos que beneficien a las sociedades futuras (Addams, 1895).

De este modo, desde la ética social (concepto desarrollado por Addams), las virtudes individuales serán preservadas en un nuevo universo moral gracias a las manifestaciones de diferentes minorías o grupos oprimidos o desvalidos, como por ejemplo el movimiento obrero o la manifestación de las mujeres, quienes buscan en la sociedad un cambio de su concepción de la verdad (Levingston, 2001), ideas que posibilitan las acciones sobre las reformas radicales o los cambios de la sociedad.

Este tipo de pragmatismo es feminista en la medida en que defiende la igualdad entre hombres y mujeres, y postula que en una sociedad democrática deben participar todos. El mutuo acuerdo y la inteligencia común eran formas de llegar a ello (Hamington, 2006).

En esta misma línea, Addams (1916) realizó una activa crítica a los sesgos de género presentes en su época, denunciando las desigualdades e injusticias cometidas en contra de las mujeres. Establecía que para el progreso social (lateral) es fundamental la diversidad de opiniones, por lo que era esencial "dar una voz" a las personas que se encontraban en situación de margina- lidad. De esta manera, promovía que las mujeres inmigrantes de la clase trabajadora tuvieran ideas relevantes para contribuir a la comunidad, las cuales debían ser escuchadas y consideradas (Hamington, 2006). Una expresión de lo anteriormente descrito fue la Hull House.

\section{Relevancia de la Hull House}

En 1889, dos mujeres, Jane Addams y Ellen Gates Starr (1859-1940) fundaron la Hull House, un asentamiento social considerado "el corazón de la reforma de mujeres en Chicago" (Quiroga, 1995, p. 37). Esta institución contribuía a la formación política, profesional y laboral de muchas mujeres de Chicago, además de brindar apoyo a muchas familias, principalmente inmigrantes, en el contexto de la era industrial (Addams, 1912b; 1916; Knight, 2005; Miranda, 2007; Kuiper, 2010)

Uno de los objetivos de la Hull House fue crear un lazo entre las mujeres y algunos varones progresistas de clase media, con los grupos de personas pobres e inmigrantes que formaban parte de la industrializada ciudad de Chicago (Addams, 1912b).

La Hull House estaba conformada completamente por mujeres progresistas de clase media, que vivían una vida alternativa al matrimonio y la familia, y que participaban de manera activa en las reformas públicas de la sociedad (Metaxas, 2000; Quiroga, 1995). A cargo de Addams, la Hull House lideró el movimiento de los asentamientos sociales en Estados Unidos, y expandió el dominio de "lo femenino", redefiniendo nuevos 
roles para miles de mujeres, quienes comenzaban a salir cada vez más de sus hogares (Wade, 2005). En 1911, existían 215 asentamientos sociales, y el 53\% eran sólo de dominio femenino (Quiroga, 1995). Esto motivaba a muchas mujeres a tomar nuevas funciones y empoderarse frente a las reformas sociales del país.

La Hull House ayudaba a la clase trabajadora de Chicago. Las mujeres estudiaban las condiciones y circunstancias en las cuales vivían sus vecinos, lo que se traducía, por un lado, en ayuda material inmediata, y por otro, en la elaboración de teorías al respecto, tarea que compartían con la Universidad de Chicago (Metaxas, 2000; Miranda, 2007).

Las mujeres de la Hull House pensaban que empleando métodos científicos para analizar las condiciones sociales, de salud, educacionales y económicas de la sociedad, se podría educar al respecto, aumentando el interés por las reformas y logrando cambios para mejorar la comunidad (Quiroga, 1995; Wade, 2005; Miranda, 2007).

El trabajo de las mujeres obreras de Chicago era apoyado por la Hull House, desde la apertura de centros diurnos para sus hijos (Quiroga, 1995), hasta su participación en las políticas públicas de la ciudad; las líderes de la Hull House procuraron ser estrategas para luchar por los derechos de la clase obrera trabajadora, por ejemplo, formando puentes entre ellas y los varones más influyentes del Estado (Knight, 2005).

Otro de los fuertes lazos que establecieron, fue con los Clubes de Mujeres, los cuales financiaban diferentes proyectos como jardines infantiles para niños en situación de pobreza y otras fuentes de empleo para mujeres de clase obrera (Quiroga, 1995).

\section{Clarke Slagle y el desarrollo de la Terapia Ocupacional}

Por sus grandes contribuciones a la profesión, Clarke Slagle fue Ilamada por sus colegas "la Jane Addams de la Terapia Ocupacional" (Quiroga, 1995; Metaxas, 2000). Una vez terminado el curso en la Escuela de Cívica y Filantropía de Chicago, Clarke Slagle trabajó en diferentes hospitales de Michigan y Nueva York, donde organizaba y posteriormente analizaba clases reeducativas para personas con enfermedad mental. Poco a poco fue creciendo el reconocimiento por su trabajo en la aplicación de ocupaciones como tratamiento y fue aumentando su contacto con los líderes del Movimiento de Higiene Mental. Su innovador método de "reeducación" la llevó a dirigir el primer departamento de Terapia Ocupacional en Henry Phipps Psychiatric Clinic of Johns Hopkins Hospital, liderado por el psiquiatra Adolf Meyer (Breines, 1986; Quiroga, 1995; Metaxas, 2000).

Mientras que Clarke Slagle trabajó con Meyer (entre 1912 y 1914) (Sanz y Rubio, 2011), visitó frecuentemente a su amigo William Rush Dunton (uno de los padres de la Terapia Ocupacional, junto a Meyer). Así, comenzaron a formular la idea de organizar una asociación que promoviera el uso de las ocupaciones como tratamiento, por lo que, siguiendo la división de roles de género de la época, Dunton se preocupó de ser portavoz y difu- 
sor por medio de artículos científicos que explicaban las relaciones teóricas entre la Terapia Ocupacional y la Psiquiatría, incluyendo los aportes teóricos de Meyer ${ }^{2}$. Simultáneamente, Clarke Slagle aumentaba su experiencia clínica y promovía la Terapia Ocupacional por medio de las redes sociales de mujeres, además realizaba diferentes consultorías a Dunton sobre ocupación en el Instituto Sheppard Pratt, (Breines, 1986; Quiroga, 1995).

Clarke Slagle mejoró de forma notable sus métodos trabajando con Meyer, incluso desarrolló el considerado primer método de intervención en Terapia Ocupacional, denominado Entrenamiento en hábitos (Kielhofner, 2006; 2009; Sanz y Rubio, 2011). Esta técnica tenía por objetivo la "reeducación" de los y las pacientes con enfermedades crónicas, buscando el desarrollo de nuevos hábitos "decentes", "dignos" y necesarios para la vida; como también, la sustitución de hábitos nocivos, para lo cual, dentro de un programa individualizado, se indagaban maneras de reemplazarlos por otros "mejores" o que fueran en beneficio de su salud (Clarke-Slagle, 1944).

En 1914, Clarke Slagle regresó a Chicago a dar una serie de conferencias sobre Terapia Ocupacional en la Escuela de Cívica y Filantropía (Hull House), y comenzó a participar a tiempo completo en la expansión de la Terapia Ocupacional y en los movimientos de reforma en la salud mental (James, 1971; Quiroga, 1995; Metaxas, 2000). En 1915 estableció la primera escuela profesional de Terapia Ocupacional de Estados Unidos: The Henry B. Favill School of Occupations, perteneciente a la Hull House, y bajo la jurisdicción de la Sociedad de Higiene Mental de Illinois (Breines, 1986, Sanz y Rubio, 2011).

En el año 1917, Clarke Slagle era superintendenta de los departamentos de Terapia Ocupacional del Estado de Illinois, tras haber sido propuesta por el Gobernador, cargo que implicaba la supervisión de todos los hospitales de ese estado (Sanz y Rubio, 2011). Ese mismo año se fundó la Sociedad Nacional para la Promoción de la Terapia Ocupacional (National Society for the Promotion of Occupational Therapy NSPOT), en la cual participaron George Edward Barton, William Rush Dunton Jr., Thomas B. Kidner, Isabel G. Newton, Susan Cox Johnson y Eleanor Clarke Slagle (Quiroga, 1995; Peloquin, 2007; Talavera, 2007; Gordon, 2008), precursores procedentes de diversas áreas del conocimiento, que tenían en común el conocer los beneficios de la ocupación utilizada como terapia, y confiar en que este saber debiese estar a cargo de profesionales formados especialmente y de manera autónoma para ello.

En 1919, Clarke Slagle fue elegida como la primera mujer presidenta de la NSPOT, mientras se celebraba la tercera reunión en la Hull House de Chicago (Talavera, 2007). En dicha reunión, Jane Addams expresó a los asistentes la importancia de la Terapia Ocupacional y la higiene mental en la transformación social (Quiroga,

\footnotetext{
${ }^{2}$ Uno de los textos más destacados de Dunton fue Reconstruction therapy, de 1919.
} 
1995). Bajo su presidencia, en 1920 , Clarke Slagle propondría el cambio de nombre de la asociación; un año más tarde, la NSPOT cambió su nombre a la actual Asociación Americana de Terapia Ocupacional (American Occupational Therapy Association -, AOTA) (Quiroga, 1995; Metaxas, 2000).

\section{Conclusión}

Addams y Clarke Slagle pertenecieron a la primera generación de mujeres que transitó entre el voluntariado y las actividades remuneradas, lo que significó que su labor se vinculase a un "trabajo" como el de los varones, lo que solo algunas décadas atrás era impensado.

Los fundamentos de Addams y Clarke Slagle se relacionaban con el rol político de las mujeres en la sociedad y con la abogacía por la igualdad de género frente a las decisiones que eran tomadas en el país. Con esa base, Clarke Slagle fue una de las fundadoras más potentes de la disciplina en el año 1917.

Este trabajo exploró sus vidas y relaciones, destacando "lo social" presente desde los inicios de la profesión, lo que nos permitiría comprender a cabalidad que la Terapia Ocupacional siempre ha tenido fundamentos teóricos vinculados a sus principios constituyentes.

Lo anterior es concordante con la diversidad de bibliografía, especialmente latinoamericana, sobre la perspectiva social de la disciplina (Da Rocha, 2008; Galheigo, 2012; Ampuero, Villegas \& Muñoz, 2014; Navarrete, Cantero, Guajardo, Sepúlveda \& Mo- runo, 2015), entendida también como Terapia Ocupacional Social (Dias, Garcez \& Esquedo, 2006; Barros, De Almeida \& Vecchia, 2007; Reis, Barros \& Uchidomari, 2010; Malfitano, Lopes, Magalhães \& Townsend, 2014, Esquerdo, Malfitano, Silva \& Borboa, 2015). Además, con las diversas propuestas internacionales, tanto desde Terapia Ocupacional como desde la Ciencia de la Ocupación, que tienen como foco central resolver problemáticas sociales (Pollard, Sakellariou, \& Kronenberg, 2009; Galvaan, 2012; Angell, 2014; Simó \& Townsend, 2015; Ramugondo \& Kronenberg, 2015).

Addams y su enfoque en la equidad social, incluyendo el género, la "clase" y las ocupaciones, fueron una piedra edificante para la Terapia Ocupacional que forjó Clarke Slagle junto con otros pioneros de la disciplina.

En el actual Paradigma Social de la Ocupación que caracteriza a la Ocupación Humana como un fenómeno complejo, sistémico, político y vinculado a la salud, la cultura, la justicia y el bienestar de las comunidades del mundo (Morrison, Olivares y Vidal, 2010), al revisar nuestros inicios como disciplina podemos identificar cómo el enfoque social de la profesión siempre ha estado presente, incluso desde antes de su "fundación inicial" en 1917. Addams, la primera filósofa pública en Estados Unidos, y Clarke Slagle, pionera en el desarrollo, fundamentación y expansión de la Terapia Ocupacional, nos dejaron un rico legado sobre cómo "lo social" es parte inherente de nuestra esencia como profesionales, desde nuestras raíces hasta nuestro quehacer actual y futuro. 


\section{Referencias}

Addams, J. (1895). The Settlement as a factor in the Labor Movement. In Addams, J. (ed.) Hull House maps and papers. NY: Thomas Y. Crowell \& Co., pp. 183-204.

Addams, J. (1902). Democracy and Social Ethics. Urbana, IL: University of Illinois Press [2002].

Addams, J. (1912a). A Modern Lear. In Elshtain, J. B. (ed.). Jane Addams and the Dream of American Democracy. NY: Basic Books [2002].

Addams, J. (1912b). Twenty years at Hull House. NY: The McMillan Company Press [2002].

Addams, J. (1916). The Long Road of Woman's Memory. Urbana, IL: University of Illinois.

Ampuero, L., Villegas, M. V. \& Muñoz, C. M. (2014). Praxis del terapeuta ocupacional y su vínculo con la participación social: el ejercicio profesional en la realidad sociosanitaria Chilena. Revista de Estudiantes de Terapia Ocupacional, 1(2): 49-62. Disponible en: http://www.reto.ubo.cl/index. php/reto/article/view/10

Angell, A. M. (2014). Occupation-centered analysis of social difference: Contributions to a socially responsive occupational science. Journal of Occupational Science, 21(2): 104-116. doi:10.1080/14427591. 2012.711230

Barros, D. D., De Almeida, M. C. \& Vecchia, T. C. (2007). Terapia ocupacional social: diversidade, cultura e saber técnico. Revista de Terapia Ocupacional da Universidade de São Paulo, 18(3): 128-134.

Breines, E. (1986). Origins and adaptations: A philosophy of practice. Lebanon, NJ: Geri- Rehab.

Da Rocha, M. H. (2008). Terapia Ocupacional. Un enfoque epistemológico y social. Santa Fe, Argentina: Universidad Nacional del Litoral.
Clarke-Slagle, E. (1934). The Occupational Therapy Programme in the State of New York. The British Journal of Psychiatry, 80, 639-649.

Clarke-Slagle, E. (1944). Syllabus for Training of Nurses in Occupational Therapy. 2d. ed. NY: State of New York Department of Mental Hygiene.

Dewey, J. (1916). Democracia y educación. 6ta ed. Madrid: Ediciones Morata [2004].

Dias, D., Garcez, M. I. \& Esquedo, R. (2006). Terapia Ocupacional Social: una perspectiva sociohistórica. En Kronenberg, F.; Simó Algado, S. y Pollard, N. (eds). Terapia Ocupacional sin Fronteras: aprendiendo del espíritu de los supervivientes. Buenos Aires: Ed. Médica Panamericana, pp. 141153.

Dunton, W. R. (1919). Reconstruction therapy. Philadelphia: Saunders.

Echeverría, J. (1998). Filosofía de la Ciencia. 2da ed. Madrid: AKAL.

Encyclopædia Britannica. (2011). Eleanor Clarke Slagle. Encyclopædia Britannica Online. [Internet] Disponible en: http://www. britannica.com/EBchecked/topic/548030/ Eleanor-Clarke-Slagle [Consultado el 22 de julio de 2015].

Esquerdo, R., Malfitano, A., Silva, C. R. \& Borboa, P. (2015). Historia, conceptos y propuestas en la terapia ocupacional social de Brasil. Revista Chilena de Terapia Ocupacional, 15(1), 73-84. doi: 10.5354/0717-5346.2015.37132

Freedman, E. (2003). No Turning Back: The History of Feminism and the Future of Women. USA: Ballantine Books.

Galheigo, S. M. (2012). Perspectiva crítica y compleja de terapia ocupacional: actividad, cotidiano, diversidad, justicia social y compromise ético. Revista Terapia Ocupacional Galicia, 5: 176-187. Disponible en: http://www.revistatog.com/mono/num5/ compromiso.pdf 
Galvaan, R. (2012). Occupational choice: The significance of socio-economic and political factors. In G. Whiteford \& C. Hocking (Eds.). Occupational science: Society, inclusion, participation (pp. 152161). Oxford, UK: Wiley-Blackwell.

Galvaan, R. (2014). The Contextually Situated Nature of Occupational Choice: Marginalised Young Adolescents' Experiences in South Africa. Journal of Occupational Science, 22(1): 39-53. doi:10.1080/1442 7591.2014 .912124

González M. \& Pérez E. (2002). Ciencia, Tecnología y Género. Revista Iberoamericana de Ciencia, Tecnología, Sociedad e Innovación. 2. Disponible en: http:// www.oei.es/revistactsi/numero2/varios2. $\underline{\mathrm{htm}}$

Gordon, D. (2008). The history of occupational therapy. En Crepeau, E, Cohn, E \& Schell, B (eds.). Willard and Spackman's Occupational Therapy. 11th Ed. Philadelphia: Lippincott Williams \& Wilkins, pp. 203-215.

Guillette, N. (1973). Terapéutica ocupacional y salud mental. En H. Willard y C. Spackman (Eds.) Terapéutica Ocupacional. 4ta Ed. Barcelona: Jims. pp. 51-130.

Haraway, D. (1991). Ciencia, cyborgs y mujeres. La reinvención de la naturaleza. España: Ed. Cátedra, Universitat de Valencia, Instituto de la mujer.

James, E. T. (1971). Notable American Women. A Biographical Dictionary. USA: Radclif College.

James, W. (1909). El significado de la verdad. 4ta. ed. Buenos Aires: Aguilar Argentina, S.A. de Ediciones, [1974] [Trad. Luís Rodríguez Aranda].

Kielhofner, G. (2009). Conceptual foundations of Occupational Therapy practice. 4th ed. Philadelphia: F. A. Davis.

Knight, L. (2005). Citizen: Jane Addams and the struggle for democracy. Chicago: The University of Chicago Press.
Kuiper, K. (2010). The 100 most influential women of all time. NY: Britannica Educational Publishing.

Malfitano, A. P. S., Lopes, R. E., Magalhães, L. \& Townsend, E. A. (2014). Social occupational therapy: Conversations about a Brazilian experience. Canadian Journal of Occupational Therapy, 81(5): 298-307. doi: 10.1177/0008417414536712

Marcos, A. (2010). Ciencia y Acción. Una filosofía práctica de la ciencia. México: Fondo de Cultura Económica.

Metaxas, V. (2000). Eleanor Clarke Slagle and Susan E. Tracy: Personal and professional identity and development of occupational therapy in progressive Era America. In Nursing history review. Official journal of american association for the history of nursing, Vol. 8: 39-70.

Miranda, M. (2007). El compromiso con la ciencia. Conocimiento y técnica en el trabajo social. Revista internacional de ciencias sociales y humanidades SOCIOTAM, XVII, 2: 9-28.

Morrison, R. (2011a). (Re)conociendo a las fundadoras y "madres" de la terapia ocupacional. Una aproximación desde los estudios feministas sobre la ciencia. Revista Terapia Ocupacional Galicia. 8, 14 [21 p.]. Disponible en: http://www.revistatog. com/num14/pdfs/original4.pdf

Morrison, R. (2011b) Eleanor Clarke Slagle. Historia y epistemología desde los estudios feministas sobre la ciencia. Cuadernos de Terapia Ocupacional da Universidade Federal de São Carlos, 19, 2, Suplemento Especial.

Morrison, R. (2014). La filosofía pragmatista en la terapia ocupacional de Eleanor Clarke Slagle. Epistemología e historia desde los estudios feministas sobre la ciencia. Tesis Doctoral. Doctorado en Lógica y Filosofía de la Ciencia, Universidad de Salamanca, España.

Morrison, R., Olivares, D. \& Vidal, D. (2011). La Filosofía de la Ocupación Hu- 
mana y el Paradigma Social de la Ocupación. Algunas reflexiones y propuestas sobre epistemologías actuales en Terapia Ocupacional y Ciencias de la Ocupación. Revista Chilena de Terapia Ocupacional, 11:2, 102-119. doi: 10.5354/07175346.2011.17785

Navarrete, E., Cantero, P., Guajardo, A., Sepúlveda, R. \& Moruno, P. (2015). Terapia Ocupacional y Exclusión Social: Hacia una praxis basada en los derechos humanos. Charleston, SC: Ed. Segismundo.

Peirce, C. S. (1907). Pragmatismo. En: Houser, N. et al. (1998). The Essential Peirce. Selected Philosophical Writings, vols. 1-2. Bloomington: Indiana University Press. Grupo de estudios Peircianos de la Univ. de Navarra. Disponible en http:// www.unav.es/gep/

Peloquin, S. (2007). Ideas directrices de los fundadores de la sociedad nacional para la promoción de la terapia ocupacional. En: Moruno Miralles P. y Talavera Valverde M. A. (Comp.). Terapia ocupacional: una perspectiva histórica. 90 años después de su creación.. Revista Terapia Ocupacional Galicia: APGTO, pp. 135-160. Disponible en: http://www.revistatog.com

Pollard, N., Sakellariou, D. \& Kronenberg, F. (2009). A Political Practice of Occupational Therapy. Philadelphia: Elservier.

Quiroga, V. A. M. (1995). Occupational therapy: The first thirty years, 1900-1930. USA: Bethesda, MD, American Occupational Therapy Association.

Ramugondo, E. L. \& Kronenberg, F. (2015). Explaining Collective Occupations from a Human Relations Perspective: Bridging the Individual-Collective Dichotomy. Journal of Occupational Science, 22(1): 3-16. doi:10.1080/14427591.2013.781920

Reis, T. D., Barros, D. D. \& Uchidomari, I. Y. (2010). A terapia ocupacional social nos congressos brasileiros (1997-2007): desafios e debates de um campo emergente. Revista de Terapia Ocupacional da Universidade de São Paulo, 21(2): 111-120.

Sanz, P. \& Rubio, C. (2011). Eleanor Clarke Slagle. Fundadora y "madre" de la terapia ocupacional. Su legado. Revista Terapia Ocupacional Galicia. 2011, 8, 13: 1-19. Disponible en: http://www.revistatog. com/num13/pdfs/historia1.pdf

Simó, S. \& Townsend, E. (2015). Eco-social occupational therapy. British Journal of Occupational Therapy, 78(3), 182-186. Doi:10.1177/0308022614561239

Talavera, M. A. (2007). El legado de Barton. En: Moruno Miralles P., Talavera Valverde M. A. (Comp.). Terapia ocupacional: una perspectiva histórica. 90 años después de su creación. Revista Terapia Ocupacional Galicia: APGTO, pp. 161-209. Disponible en: http://www.revistatog.com

Townsend, E. \& Wilcock, A.A. (2004). Occupational justice and client-centred practice: A dialogue in progress. Canadian JOurnal of Occupational Therapy, 71, 75-87.

Wade, L. C. (2005). Settlement Houses. En: Reiff, J., Keating, A. \& Grossman, J. (Eds.). The Electronic Encyclopedia of Chicago. Chicago Historical Society. Disponible en http://www.encyclopedia.chicagohistory. org/pages/1135.html [Consultado el 13 de julio de 2015] 